\title{
PLANEJAMENTO DE PRODUÇÃO EM PROBLEMAS MULTI- PRODUTOS E MULTI-MÁQUINA̧S VISANDO À MAXIMIZAÇÃO DO DESEMPENHO ECONÔMICO*
}

\author{
PRODUCTION PLANNING MULTI-PRODUCT AND MULTI-MACHINE \\ PROBLEMS IN ORDER TO MAXIMIZE THE ECONOMIC PERFOMANCE
}

\author{
Fernanda Romanzini ${ }^{1, \Downarrow}$, José Luis Duarte ${ }^{2}$, Rodrigo Pessotto Almeida ${ }^{3}$
}

\section{RESUMO}

Este artigo propõe um novo método que visa à maximização do resultado econômico operacional, através do planejamento de produção. O modelo utiliza um algoritmo de otimização que envolve quatro áreas de análise: mercado, produção, custos e por fim o resultado econômico. O objetivo é a maximização do lucro. A aplicação do modelo ocorre em uma empresa de manufatura que tem se caracterizado por alta variabilidade na definição dos preços de venda. Os resultados deste estudo indicam que pequenas variações nos preços de venda podem comprometer substancialmente os lucros globais do sistema produtivo.

Palavras Chave: Planejamento de produção, elasticidade preço-demanda, capacidade finita; programação matemática.

\section{ABSTRACT}

This paper proposes a new method for maximizing the economic operational profit through production planning. The model uses an optimization algorithm involving four areas of analysis: market, production, costs and economic results. The objective function comprises profit maximization. The application of the model occurs in a manufacturing company that has

\footnotetext{
*Versão originalmente enviada no IX Simposio Internacional de Ingeniería Industrial: Actualidad y Nuevas Tendencias 2016-Brasil e melhorada após o processo de revisão por pares da revista Ingeniería Industrial.

1 Universidade Federal do Rio Grande do Sul, Porto Alegre, Brazil, ORCID 0000-0001-5532-3948.

${ }^{2}$ Universidade Federal do Rio Grande do Sul, Porto Alegre, Brazil, ribeiro@producao.ufrgs.br. ORCID 0000-00025795-4468.

${ }^{3}$ Universidade Federal do Rio Grande do Sul, Porto Alegre, Brazil, rodrigopa7@hotmail.com. ORCID 0000-0002-81034960 .
}

•Autor para correspondência: feromanzini@outlook.com 
been characterized by high variability in selling prices. The results, this study, indicate that small deviations in sale prices may severely reduce production system global profit.

Keywords: Production planning, price easticity of demand, finite capacity, mathematical programming.

\section{INTRODUÇÃO}

Ao relacionar assuntos envolvendo capacidade produtiva, estoques, produção e previsão de vendas, as empresas enfrentam futuros incertos, que dependem das condições de mercado, tais como: demanda, preço e custos associados a cada produto (Lingitz et al., 2013; Kassel e Tittmann, 2007). Para lidar com as incertezas envolvidas, as empresas precisam buscar ferramentas apropriadas à sua realidade, que permitam planejar e programar a produção considerando as variações que ocorrem, tanto no ambiente externo, como interno (Chod e Rudi, 2006; Kaplan et al., 2010).

Para diminuir seu custo operacional, prestar um serviço satisfatório aos seus clientes e sobreviver em um mercado globalmente competitivo, as organizações buscam continuamente a inovação em seus processos de decisão. Para isso, as organizações precisam relacionar as informações referentes à capacidade produtiva, estoques, produção, previsão de demanda, demanda e preço em um único sistema (Shapiro, 2004). Adicionalmente, para Zhang et al. (2012), esse sistema deve possuir recursos para analisar essas informações e definir a quantidade a ser produzida, visando o resultado operacional ótimo para a organização.

Conforme Biller et al., (2006), decisões estratégicas, normalmente, são baseadas em previsões de demanda, utilizando planos de produção mais sofisticados que contemplam métodos para determinar o erro de previsão de demanda, através de distribuições em torno de um ponto da previsão ou um conjunto de possíveis cenários futuros. Para atenuar o impacto dos erros de previsão, é possível utilizar conceitos de capacidade flexível. Esta é muitas vezes instalada em empresas com mix de produtos diversificado, para combater cenários onde as incertezas de demanda são elevadas (Tan e Alp, 2009; Moratewetz e Sihn, 2012; Lingitz et al., 2013).

Outra forma de tratar as incertezas da demanda é utilizando os conceitos de elasticidade preço-demanda. Conforme Liu et al. (2012), a estratégia de preços é uma questão importante para a cadeia de suprimentos, especialmente quando a elasticidade preço-demanda é alta e o preço tem um efeito significativo sobre as exigências do produto. As decisões sobre os preços corretos a serem praticados são cruciais na gestão da cadeia de fornecimento (Antunes et al., 2008). Embora haja alguns trabalhos na literatura que abordam a elasticidade preço-demanda junto a cadeias de fornecimento, nenhuma das obras citadas considera as flutuações de preços, que é uma das principais razões para o efeito chicote na cadeia de fornecimento (Liu et al., 2012).

Conforme Kannegiesser et al. (2009), para otimizar o plano de produção, pode-se utilizar modelos que visem a maximização do resultado econômico operacional da organização. Ainda para o autor, modelos de otimização podem buscar maximizar o lucro através da coordenação de volume de vendas e os respectivos preços, bem como as decisões sobre capacidade produtiva. Neste sentido, Martínez-Costa et al. (2014) afirmam que modelos desse tipo podem contribuir para equacionar os problemas associados à capacidade produtiva e 
incertezas na demanda, oferecendo soluções apropriadas para cenários de interesse. Ji et al. (2016) desenvolvem uma modelagem matemática para estabelecer a minimização dos custos de produção, considerando efeitos de variabilidade da demanda e incerteza na capacidade de produção.

Neste artigo, o tema planejamento de produção é abordado juntamente com os assuntos elasticidade preço-demanda, previsão de demanda tratada de forma estocástica e otimização de mix de produção. O objetivo principal é desenvolver um modelo que visa entender todos estes assuntos abordados e resolver um problema enfrentado em muitas organizações. O resultado esperado é definir um mix ótimo de produção associado a preços de venda ótimos para um conjunto de produtos, respeitando a capacidade fabril e levando em consideração os erros de previsão de demanda.

\section{Elasticidade preço-demanda aplicada ao planejamento de produção}

Segundo Botelho (2005), a elasticidade preço-demanda é o conceito que determina a relação entre o preço do produto e a sua respectiva demanda. Segundo este autor, esse conceito é usado para medir o grau de resposta da demanda quando houver alteração no preço. Os índices de elasticidades preço-demanda são quase sempre negativos, seguindo a lei da demanda, que determina que uma redução no preço do produto leva a um aumento na demanda do produto, e vice-versa (embora o índice possa ser positivo em alguns casos especiais). O coeficiente de elasticidade preço-demanda de cada produto em cada mercado é definido como a variação percentual na quantidade do produto demandada pela variação percentual no preço (Liu et al., 2012; Lanning et al., 2000; Botelho, 2005).

Kaplan et al. (2010) afirmam que a relação entre preço de venda e a demanda de um produto é estabelecida através da curva de elasticidade preço-demanda, onde a demanda surge como uma função não linear do preço estabelecido. Na cadeia de suprimentos, muitas vezes, esta relação é omitida no nível tático. No entanto, independentemente da curva ser ou não explicitada, o preço invariavelmente é um dos parâmetros mais importantes na determinação da quantidade demandada, conforme Tellis (1988) já afirmava.

Segundo Chod \& Rudi (2006), elasticidade preço-demanda pode sofrer alterações quando há venda em diferentes mercados geográficos ou diferentes segmentos de mercado, ou, ainda, quando são comercializados produtos com maior diferenciação em relação a seus concorrentes. Sendo assim, as empresas investem seus recursos com base em previsões de mercados imperfeitos e, para obter as funções gerais de lucro, são considerados cenários com elasticidade preço-demanda constantes. Nestes casos, é possível prever os cenários de 
demanda considerando as alterações de preços praticadas nos respectivos mercados.

Para maximizar os lucros operacionais, Jans et al., (2008) apresentam um modelo que visa à integração de informações de produção, de marketing e contábeis. O modelo desenvolvido incorpora programação matemática, com o objetivo de reduzir custos e maximizar o desenvolvimento de produtos. A formulação do problema tem como base uma decisão de investimento utilizando os conceitos de VPL (Valor Presente Líquido).

Kaplan et al. (2010) utilizam um modelo de otimização para integração da cadeia de suprimentos multiprodutos. O modelo proposto por esses autores inclui a elasticidade preçodemanda. Neste modelo a elasticidade preço-demanda pode ser considerada um valor fixo ou um valor variável ao longo do período. O preço de venda de um produto é dado a partir de informações da demanda e elasticidade de períodos anteriores ao plano atual. Além disso, ao utilizar os dados de elasticidade de períodos anteriores, deve-se presumir que os valores de elasticidade preço-demanda de um produto permaneçam estáveis durante sua vida (Kaplan et al., 2010).

\section{Processo de Planejamento e Otimização da Produção}

Atualmente, as empresas operam com objetivos de negócio diferentes. Algumas, por exemplo, definem a maximização do resultado como seu principal objetivo final. Outras buscam aumentar sua participação no mercado ou alcançar maiores níveis de satisfação dos clientes. Todos estes pontos podem ser alcançados através de estratégias de preços bem desenvolvidas, que devem considerar três elementos básicos, custos, clientes e concorrência (Danese \& Kalchschimidt, 2011; Fischer \& Raman, 1996). Ações para obter uma gestão de custos integrada, um conhecimento aprofundado sobre o comportamento do cliente e uma noção real sobre concorrência de mercado em uma estrutura unificada são a chave para o desenvolvimento de uma estratégia de preços e, consequentemente, a maximização do resultado operacional (Levis \& Papageorgiou, 2007). Nesse sentido, conforme realça Cezarino (2007), o plano de produção para empresas de manufatura assume papel fundamental no processo de planejamento e construção de metas estratégicas. Danese \& Kalchschmidt (2011) investigam os impactos da acuracidade das previsões e sua forma de condução sobre o desempenho operacional relacionado a custos de produção e entrega. Para estes a estrutura do processo de previsão deve ser coerente com a forma como os gestores pretendem aproveitar os resultados previstos, e com o objetivo que deve ser alcançado.

Recentemente, Subramanian et al. (2013) propuseram a utilização do controle preditivo por modelos (MPC - Model Predictive Control), para a otimização da cadeia de suprimentos, especificamente voltado à gestão de estoques. Modelos dinâmicos são utilizados para prever resultados futuros otimizados, onde estes consideram as restrições e interações presentes no sistema. Baseado nos resultados previstos, esta abordagem permite identificar as melhores decisões operacionais para viabilizar o alcance do melhor resultado prático. Adicionalmente, o modelo proposto apresenta um conceito cooperativo, onde cada agente da cadeia toma decisões locais, porém o objetivo é voltado à otimização global da cadeia de suprimentos. Assim o objetivo do controle neste modelo foi de minimizar desvios dos níveis de estoque. Porém, os autores apontam a necessidade de implementar o MPC com variáveis de controle econômicas, uma vez que na prática a gestão da cadeia de suprimentos busca a otimização do desempenho econômico. 


\section{Modelo}

Esta pesquisa, em sua natureza, classifica-se como aplicada, pois está focada na solução de um problema prático, envolvendo a integração de aspectos associados à elasticidade preço-demanda, previsão de demanda tratada de forma estocástica e otimização de mix de produção. A abordagem da pesquisa é predominantemente quantitativa, pois se apoia na base de dados de uma empresa e em fórmulas matemáticas. Quanto aos objetivos, esta pesquisa classifica-se como explicativa, onde a ênfase é a construção de um modelo para planejar a produção considerando a elasticidade preço-demanda e a capacidade produtiva. O procedimento da pesquisa enquadra-se como uma pesquisa-ação, pois os autores estiveram diretamente envolvidos no estudo e implementação da solução em um problema prático (Silva \& Menezes, 2001).

Para desenvolvimento do método proposto, inicialmente foi realizada uma pesquisa na literatura com os temas relacionados ao estudo. Sendo, os assuntos abordados, a elasticidade preço-demanda aplicada ao planejamento de produção e o processo de planejamento e otimização da produção associados. Na construção do método, buscou-se relacionar os conceitos teóricos com as principais características envolvidas na aplicação prática, visando maximizar o lucro da operação empresarial. A Tabela 1 apresenta um resumo dos principais autores pesquisados, com o objetivo de realizar a comparação deste modelo com o que já está sendo apresentado na literatura.

A fim de entender o ambiente modelado, as principais premissas são elencadas: (i) a distribuição dos preços dos concorrentes mantém-se aproximadamente constante, pois há vários concorrentes, alguns podem reduzir o preço em um determinado mês, mas isso é aproximadamente compensado pelo aumento de preços que outros concorrentes aplicam no mês em questão; (ii) trata-se de um produto da construção civil, que pode ser usado tanto em novas construções como em reformas. Devido a esse aspecto, o mercado desse produto é pouco afetado por crises econômicas (considerando curto e médio prazo), de forma que o tamanho de mercado, no horizonte das previsões realizadas, pode ser considerado relativamente estável; (iii) o interesse é a maximização do lucro; outras considerações de caráter estratégico não são contempladas no modelo.

Tabela 1. Resumo dos principais autores

\begin{tabular}{|l|l|}
\hline \multicolumn{1}{|c|}{ Elaborado por: } & \multicolumn{1}{c|}{ Modelos apresentados: } \\
\hline Almeida et al.(2010) & Método calcula a elasticidade preço-demanda, através do MQO. \\
\hline Biller et al. (2006) & $\begin{array}{l}\text { Modelo avalia investimentos na capacidade produtiva flexível } \\
\text { ou dedicada, através da determinação do preço de venda em } \\
\text { diferentes cenários da demanda. }\end{array}$ \\
\hline Bose \& Pekny (2000) & $\begin{array}{l}\text { Previsão e otimização da cadeia da cadeia de suprimentos, } \\
\text { através do Controle Preditivo por Modelos (CPM). }\end{array}$ \\
\hline Botelho (2005) & $\begin{array}{l}\text { Modelo calcula o índice de elasticidade preço-demanda, através } \\
\text { de dois parâmetros: escolha da marca e quantidade comprada }\end{array}$ \\
\hline Chod \& Rudi (2006) & $\begin{array}{l}\text { Modelo maximiza o lucro, em ambientes de investimentos de } \\
\text { recursos, com base em previsões de mercados incertos. Tem } \\
\text { como base elasticidade preço-demanda com índice constante. }\end{array}$ \\
\hline $\begin{array}{l}\text { Erdirik-Dogan \& } \\
\text { Grossmann (2008) }\end{array}$ & $\begin{array}{l}\text { Modelo maximiza lucros, relacionando vendas e custos, em dois } \\
\text { níveis, planejamento e programação da produção. }\end{array}$ \\
\hline
\end{tabular}




\begin{tabular}{|c|c|}
\hline Hsieh et al., (2010) & $\begin{array}{l}\text { Investiga as principais influências provocadas pela elasticidade } \\
\text { preço-demanda, através das variações no preço. }\end{array}$ \\
\hline Jans et al. (2008) & $\begin{array}{l}\text { Modelo maximiza lucros em um cenário de análise de } \\
\text { investimentos, considerando custos, receitas, elasticidade } \\
\text { preço-demanda como variáveis de decisão. }\end{array}$ \\
\hline $\begin{array}{l}\text { Kannegiesser et } \\
\text { al.(2009) }\end{array}$ & $\begin{array}{l}\text { Modelo integrado de otimização de resultados, tendo como } \\
\text { principais variáveis o preço de venda e lotes de produção. }\end{array}$ \\
\hline Kaplan et al. (2010) & $\begin{array}{l}\text { Modelo visa à otimização da cadeia, através dos efeitos da } \\
\text { elasticidade preço-demanda nas decisões de produção e } \\
\text { estoque. }\end{array}$ \\
\hline Kim \& Lee (1998) & $\begin{array}{l}\text { Modelo aplica programação geométrica, com o objetivo de } \\
\text { maximizar o lucro e definir o preço ideal, para dimensionar a } \\
\text { capacidade produtiva fixa e variável. }\end{array}$ \\
\hline Papageorgiou (2009) & $\begin{array}{l}\text { O estudo concentra em uma revisão de modelos de } \\
\text { programação matemática para problemas de otimização da } \\
\text { cadeia de suprimentos na indústria de processo, que podem ser } \\
\text { aplicados tanto no plano estratégico, quanto no plano tático. }\end{array}$ \\
\hline Leung (2007) & $\begin{array}{l}\text { Modelo utiliza programação geométrica, para determinar lotes } \\
\text { econômicos de produção, considerando flexibilidade e custos. }\end{array}$ \\
\hline $\begin{array}{l}\text { Levis \& Papageorgiou } \\
\text { (2007) }\end{array}$ & $\begin{array}{l}\text { Modelo determina políticas de preços ótimas, tendo em } \\
\text { consideração: a demanda dos produtos, custos de produção, } \\
\text { disponibilidade de recursos, elasticidade preço-demanda, } \\
\text { terceirização e concorrência. }\end{array}$ \\
\hline Perea-Lo et al. (2003) & $\begin{array}{l}\text { O modelo maximiza lucro e o nível de serviço da cadeia de } \\
\text { suprimentos, considerando as variáveis: receita e custos } \\
\text { diversos. }\end{array}$ \\
\hline Sadjadi et al. (2012) & $\begin{array}{l}\text { Modelo utiliza programação geométrica para a maximização } \\
\text { dos lucros, através do dimensionamento ideal de lotes, preços, } \\
\text { flexibilidade e confiabilidade do processo de produção. }\end{array}$ \\
\hline Sen et al. (2004) & $\begin{array}{l}\text { Modelo visa à otimização da cadeia de suprimentos, } \\
\text { considerando: ciclo de vida do produto, alteração no preço, nível } \\
\text { de serviço prestado e lucratividade. }\end{array}$ \\
\hline Shapiro (2004) & $\begin{array}{l}\text { Estudo investigativo sobre os desafios da aplicação da } \\
\text { modelagem com análise quantitativa dos problemas de } \\
\text { planejamento na cadeia de suprimentos. }\end{array}$ \\
\hline Liu et al. (2012) & $\begin{array}{l}\text { Modelo para planejamento e maximização do lucro na cadeia de } \\
\text { suprimentos, considerando incertezas na demanda, flutuações } \\
\text { de preço e níveis de estoque. }\end{array}$ \\
\hline $\begin{array}{l}\text { Subramanian et al. } \\
(2013)\end{array}$ & $\begin{array}{l}\text { Modelo utiliza MPC, para otimização da cadeia de suprimentos, } \\
\text { com objetivo de minimizar estoques. }\end{array}$ \\
\hline $\begin{array}{l}\text { Viswanathan et al. } \\
\text { (2003) }\end{array}$ & $\begin{array}{l}\text { Modelo maximiza lucros, através de políticas de descontos, em } \\
\text { ambientes com demanda sensível ao preço de venda praticado. }\end{array}$ \\
\hline
\end{tabular}


Para melhor entender o método utilizado neste artigo, foi criado um esquema que procura relacionar os temas abordados e as principais etapas que compõem a estrutura do modelo desenvolvido. Num primeiro momento procurou-se identificar as principais áreas que estão envolvidas, sendo elas: mercado, produção e custos. Sendo assim, é possível entender que a união das informações obtidas, em cada área, é responsável pela composição do resultado deste modelo, conforme está representado na figura 1.

MERCADO

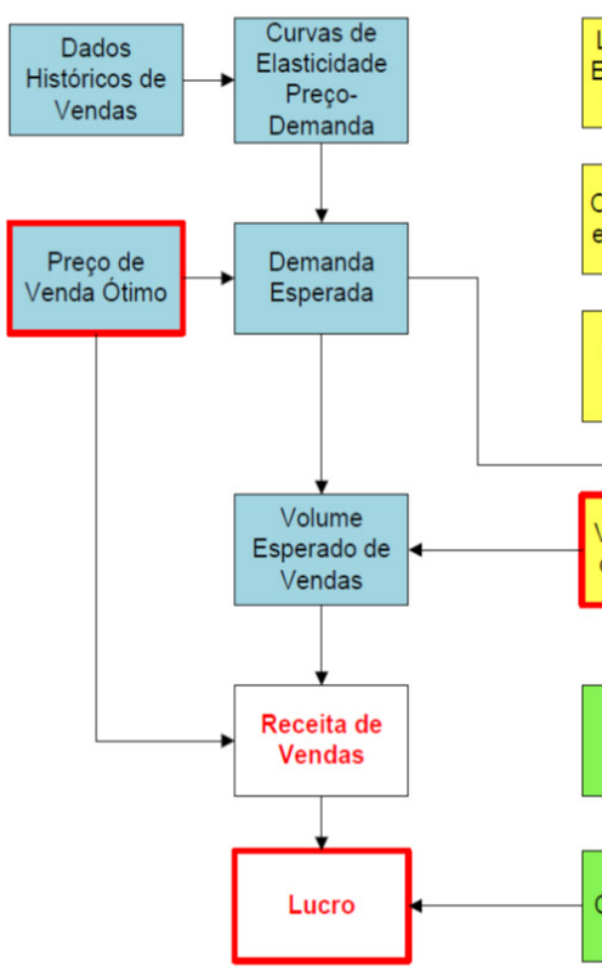

RESULTADOS

\section{PRODUCCÃO}

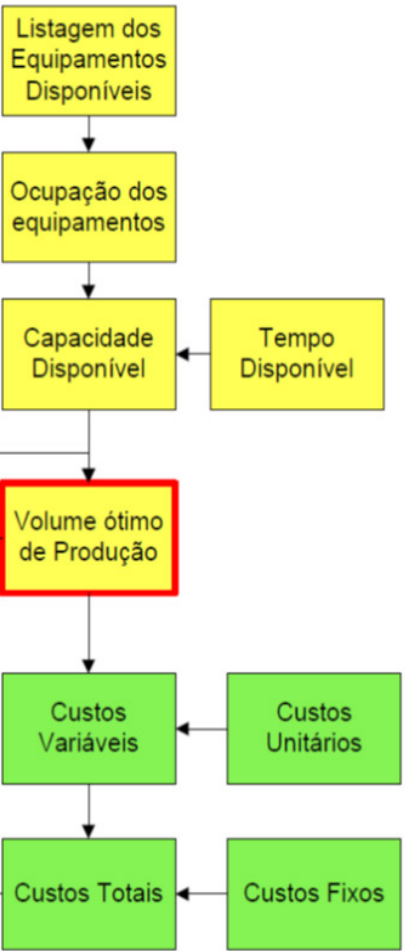

CUSTOS

Figura 1. Etapas do modelo proposto.

Fonte: Desenvolvido pelos autores.

No bloco de mercado, relacionam-se as informações do ambiente comercial. Nesta etapa, são obtidos os dados históricos de preço e demanda que são utilizados como base para compor as curvas de elasticidade preço-demanda. Estas, por sua vez, são utilizadas para obter a demanda esperada para o período, juntamente com o preço ótimo, que é obtido através de programação matemática. Calculado o volume esperado da demanda, este é associado ao volume ótimo de produção, que juntos são responsáveis por gerar a informação das vendas esperadas no período. No bloco de produção, inicialmente, parte-se da listagem dos equipamentos disponíveis, que na sequência são alocados para os produtos em análise. Esta informação, juntamente com o tempo, em horas disponíveis, serve como base para o cálculo da capacidade produtiva disponível. Tendo conhecimento da capacidade produtiva disponível, e o volume esperado da demanda para o período, citado anteriormente, é possível obter o volume ótimo de produção, que também é definido via programação matemática. 
No bloco de custos, são obtidas as informações sobre os custos de fabricação unitários; estes, multiplicados às quantidades a serem produzidas, compõem os custos variáveis, juntamente com o custo de estocagem, que é considerado apenas quando o produto é estocado. Os custos fixos também são levantados. No entanto, de forma simplificada, estes são rateados entre as quantidades produzidas para que sejam somados aos custos variáveis unitários e, assim, o custo total do produto é obtido. No bloco resultados ocorre à maximização do lucro, que depende basicamente da receita obtida e dos custos totais de fabricação. A receita é calculada através da multiplicação entre o preço ótimo, estabelecido pela programação matemática, e o volume esperado de vendas. No decorrer desta Seção, as etapas citadas e o formulário utilizado são apresentados detalhadamente.

Utilizando os dados históricos de vendas mensais, é possível obter as curvas de elasticidade preço-demanda de cada item auxiliando, assim, na previsão da demanda média em função do preço a ser definido. Após testar diferentes ajustes, a equação 1 forneceu melhores resultados e foi utilizada para calcular o valor esperado da demanda $(\hat{D})$ do período.

$$
\widehat{D}_{i}=M_{i} \times\left(-\left(\left(\frac{P V_{i}-L_{i}}{A_{i}}\right)^{B_{i}}\right)\right)
$$

Onde $i$ refere-se ao produto ( $i$ é igual de 1 a $n, n$ são os produtos fabricados e vendidos). $M_{i}$ é o tamanho total do mercado para o produto i, representado pela unidade quilogramas $(\mathrm{kg})$, que seria conquistado se o preço fosse igual ou inferior a $L_{i}$; $L_{i}$ é um preço mínimo, dado pela unidade real por quilograma $(R \$ / \mathrm{kg})$, que conduziria a conquistar todo o mercado; enquanto $A_{i}$ e $B_{i}$ são os parâmetros que controlam a inclinação e curvatura da elasticidade preço-demanda. A partir dos valores $M_{i}$ e $L_{i}$ já informados pelos especialistas da empresa, as constantes $A_{i}$ e $B_{i}$ foram definidas, para cada produto, usando rotinas de regressão nãolinear. $\mathrm{O} P V_{i}$ é o preço ótimo de venda $(R \$ / \mathrm{kg})$, obtido através da programação matemática, utilizando o algoritmo GRG (Gradiente Reduzido Generalizado) não linear e adotando como função objetivo maximizar o Lucro.

A próxima etapa, no bloco de Mercado, contempla o cálculo do Volume Esperado de Vendas $\left(\hat{V}_{i}\right)$ que pode ser calculado usando conceitos probabilísticos. Nesse estudo, para representar a distribuição de probabilidade da demanda $f(x)$, conforme ilustrado na figura 2, qualquer distribuição de demanda pode ser adotada para lidar com casos diversos. A variabilidade da demanda, também foi calculada, e é representada por $\sigma_{i}$.

Na figura 2, A1 é representada pela equação 2, e $A_{2}$ pela equação 3. A estimativa de vendas é fornecida pela equação 4 .

$$
\begin{gathered}
A_{1}=\int_{0}^{P_{i}} f_{i}(x) d x \\
A_{2}=\int_{P_{i}}^{\infty} f_{i}(x) d x \text { ou } A_{2}=1-A_{1} \\
\widehat{V}_{l}=\int_{0}^{P_{i}} x f_{i}(x) d x+A_{2} P_{i}
\end{gathered}
$$


Onde $\widehat{V}_{i}$ corresponde à estimativa das vendas para o produto $i ; x$ é a demanda; $f(x)$ é a distribuição de probabilidade da demanda para o produto $i$, e $P_{i}$ corresponde ao valor ótimo de produção para o produto $i$, que conduz à maximização do lucro, respeitando as restrições de produção.

No bloco que envolve a Produção, inicia-se com a elaboração de listagem dos equipamentos disponíveis com suas respectivas restrições, capacidades e flexibilidades. Na sequência é definida a ocupação dos equipamentos, considerando as restrições dos produtos em estudo. Assim, cada produto irá ocupar um número de horas de cada equipamento.

Nesta etapa, são levantados dados referentes à produtividade de cada máquina, através do tempo necessário para a produção de cada produto do mix em estudo. Logo, fica estabelecida a capacidade fabril, que tem como principal função no modelo estabelecer o limite da quantidade a ser produzida, através do tempo disponível, pois trata-se de um modelo desenvolvido para lidar com plantas fabris com capacidade finita.

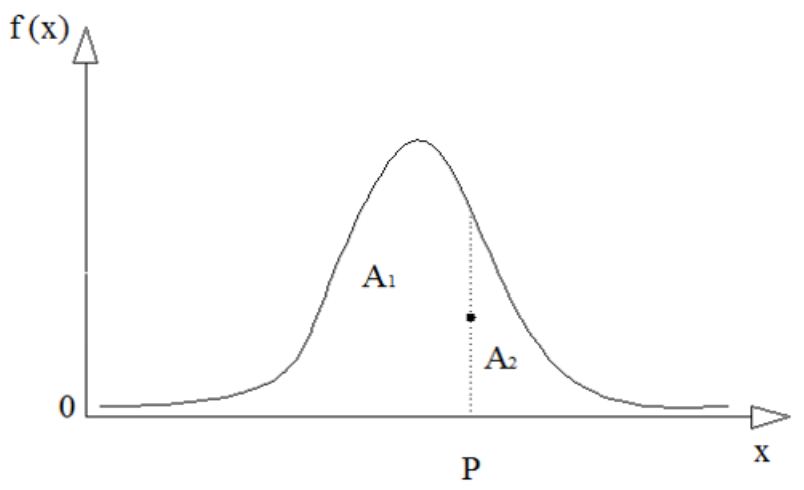

Figura 2. Curva de distribuição, utilizada para representar a demanda.

Fonte: Desenvolvido pelos autores.

O cálculo dos volumes ótimos de produção $\left(P_{i}\right)$ é obtido através de programação matemática, considerando a capacidade disponível e a previsão de demanda para o período. Logo, conforme explicado anteriormente, usando programação matemática definem-se os preços ótimos e volumes ótimos, visando maximizar o Lucro.

O valor esperado do Estoque para o produto $i\left(E_{i}\right)$ corresponde à diferença entre o valor esperado das vendas e o volume ótimo de produção, conforme equação 5 .

$$
\widehat{E}_{\imath}=P_{i}-\widehat{V}_{\imath} \quad \text { (eq. 5) }
$$

O modelo também envolve os custos gerais da operação, representado no terceiro bloco da figura 1. Para isso são levantados os custos unitários variáveis $\left(C U V_{i}\right)$ de fabricação e os custos fixos do sistema produtivo. Os custos variáveis unitários $\left(C V_{i}\right)$ dependem do volume a ser produzido, conforme a equação 6 . 


$$
C V_{i}=P_{i} \times C U V_{i}+\widehat{E}_{i} \times C U E_{i} \quad \text { (eq. 6) }
$$

Os custos fixos, nesse estudo, são rateados proporcionalmente ao custo variável, os quais dependem da quantidade produzida e fornecem uma indicação do esforço mobilizado na produção de cada produto, onde $C F_{i}$ é o custo fixo do produto $i, C V_{i}$ é o custo variável do produto $i, C V_{T}$ é o custo variável total e $C F_{\mathrm{T}}$ é o custo fixo total e estão representados na equação 7.

$$
C F_{i}=\frac{C V_{i}}{C V_{T}} \times C F_{T} \quad(\text { eq. } 7)
$$

Então o custo total de cada produto $i$ é calculado como o somatório do custo variável total e o custo fixos de $i$ rateados. Logo, o custo total é o somatório dos custos totais de cada produto $i$, conforme equações 8 e 9 , respectivamente.

$$
\begin{gathered}
C T_{i}=C V_{i}+C F_{i} \quad \text { (eq. 8) } \\
C T=\sum C T_{i} \quad \text { (eq. 9) }
\end{gathered}
$$

A etapa dos resultados visa à otimização do Lucro Total (î́) do sistema, que por sua vez, depende diretamente das receitas das vendas e dos custos totais, conforme ilustrado na equação 10. As receitas das vendas são calculadas multiplicando os preços definidos para cada produto pelos volumes de vendas calculados para cada produto. O lucro depende tanto das receitas de vendas como dos custos totais, sendo calculado proporcionalmente ao Volume esperado de Vendas $\left(\hat{V}_{i}\right)$.

$$
\widehat{L}=\sum\left(P V_{i} \times \widehat{V}_{l}\right)-\sum C T_{i} \quad(\text { eq. 10) }
$$

\section{ESTUDO APLICADO E DISCUSSÕES}

Após a apresentação do modelo, buscou-se realizar a aplicação do mesmo. Nesta seção são apresentados os resultados da aplicação, que ocorreu em uma empresa fabricante de materiais em PVC para a construção civil. Os produtos abordados são utilizados nos acabamentos internos de residências e lojas. A empresa possui atuação nacional na comercialização de seus produtos. No entanto, a empresa observa variabilidade na demanda em decorrência da instabilidade na definição de seus preços de venda, pois a mesma não utiliza métodos formais de otimização de preços.

A aplicação do método inicia com a coleta dos dados na empresa em estudo. Três áreas da 
empresa foram consultadas: comercial, com os históricos de vendas e preços praticados; contabilidade, com as informações de custos gerais; e industrial, com as informações sobre capacidade produtiva.

Através dos dados históricos de venda, as curvas de elasticidade preço-demanda foram modeladas em função dos preços e volumes comercializados no período analisado. Isso foi feito aplicando a equação 1, que fornece a expressão da demanda para cada produto em análise. As informações sobre o mercado (variáveis $M$ e $L$ ) foram obtidas em conversas com especialistas da área comercial, que possuem experiência neste mercado a mais de 20 anos. Logo, o cálculo de previsão de demanda, ocorre em duas etapas. A primeira envolve a modelagem das curvas de elasticidade preço-demanda. Já a segunda etapa envolve a previsão da demanda propriamente dita, que depende do preço estabelecido. Conforme explicado no modelo, outras variáveis estão envolvidas neste cálculo e são apresentadas na sequência. Tabela 3, mostra os resultados obtidos nesta etapa.

A próxima etapa no bloco de Mercado envolveu o cálculo do Volume Esperado de Vendas $\left(\hat{V}_{i}\right)$ para cada produto analisado. Nesse estudo, foi adotada uma distribuição triangular para representar a probabilidade da demanda $f(x)$, conforme ilustrado na figura 3 . No entanto, qualquer outra distribuição de demanda poderia ser adotada para lidar com casos diversos. Os limites da distribuição triangular foram definidos conforme a variabilidade natural das vendas, extraída da análise do histórico de vendas. Os limites adotados foram estabelecidos como dois desvios padrões acima e abaixo do valor esperado da demanda.

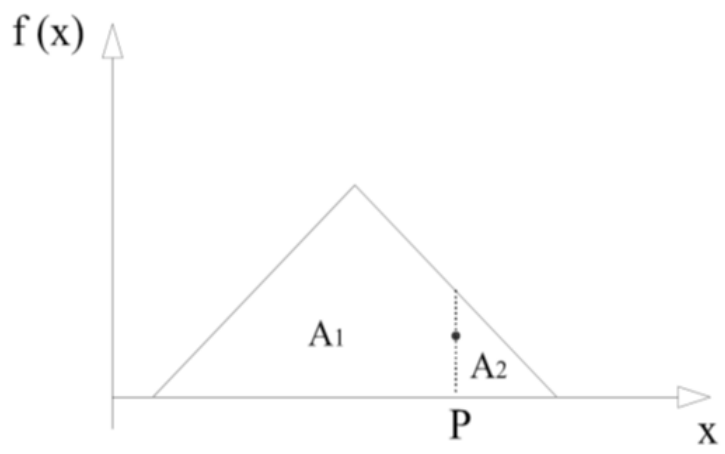

Figura 3 : Curva de distribuição triangular, utilizada para representar a demanda. Fonte: Desenvolvido pelos autores.

Em paralelo à resolução das variáveis do bloco de mercado, foram levantados os dados referentes à área produtiva. A listagem dos equipamentos, com suas respectivas produtividades, segue na Tabela 2, conforme informado pela empresa.

Tabela 2. Produtividade de Máquina

\begin{tabular}{ccccl}
\hline Produto & P1 & P2 & P3 & Unidade \\
\hline Produtividade da Máquina 1 & 550 & & 100 & $\mathrm{Kg} / \mathrm{hora}$ \\
\hline Produtividade da Máquina 2 & & 360 & 200 & $\mathrm{Kg} / \mathrm{hora}$ \\
\hline Produtividade da Máquina 3 & & 360 & & $\mathrm{Kg} / \mathrm{hora}$ \\
\hline Produtividade da Máquina 4 & & 360 & & $\mathrm{Kg} / \mathrm{hora}$ \\
\hline
\end{tabular}


É possível observar que o produto $\mathrm{P} 1$ ocupa apenas a máquina 1 na sua fabricação. Já o produto P2 ocupa as máquinas 2, 3 e 4, enquanto o produto P3 ocupa as máquinas 1 e 2 . Os produtos abordados possuem formatos e dimensões diferentes, por isso, as diferentes produtividades em um mesmo equipamento.

O fator limitante, neste estudo são as horas disponíveis para cada equipamento, que neste estudo são 380 horas mensais. Logo, é possível calcular a capacidade de produção de cada item.

Apesar de ser apresentado um modelo segregado em blocos, a aplicação do modelo é feita de maneira integrada. Na primeira etapa, conforme mencionado acima, aplica-se a equação 1 , em que o preço de venda é uma variável a ser ajustada. Paralelamente, os volumes ótimos de produção $\left(P_{i}\right)$ são ajustados, também via otimização matemática, considerando a capacidade fabril e a demanda esperada, sendo que o ajuste de preços e volumes tem como objetivo maximizar o lucro do sistema.

Após pesquisa na área de contabilidade da empresa, as informações reais sobre os custos foram obtidas. Logo, aplicaram-se as funções propostas no modelo, e obtiveram-se os resultados apresentados na Tabela 3.

A Tabela 3 apresenta um resumo dos cálculos realizados. Os dados informados ao sistema estão apresentados na cor azul, as variáveis ajustáveis a serem otimizadas estão em vermelho, e a função objetivo a ser maximizada está em amarelo. Os demais dados são calculados conforme as equações apresentadas no modelo.

O modelo apresentado neste estudo contempla a relação entre importantes áreas das organizações: comercial, produção e contabilidade. Ele trabalha as informações geradas em cada área de forma integrada. A aplicação deste modelo é de fácil entendimento e aplicação, no entanto requer a compilação de vários dados, logo é preciso que as áreas envolvidas possuam registro e acompanhamento das informações necessárias.

Ao comparar este modelo com os temas abordados pelos autores pesquisados no referencial teórico deste artigo, percebe-se que os assuntos são tratados pela literatura, de forma independente, ou não abordam todos os aspectos ressaltados neste estudo. Sendo assim, este modelo diferencia-se dos demais citados por apresentar um conjunto de informações de mercado associadas às curvas de elasticidade preço-demanda, à otimização da capacidade produtiva disponível e aos custos de produção, fixos e variáveis. O objetivo é otimizar preço de venda e volume de produção, visando à maximização do resultado econômico, o lucro, além de definir informações importantes ao tomador de decisão, tais como o volume esperado de venda. 


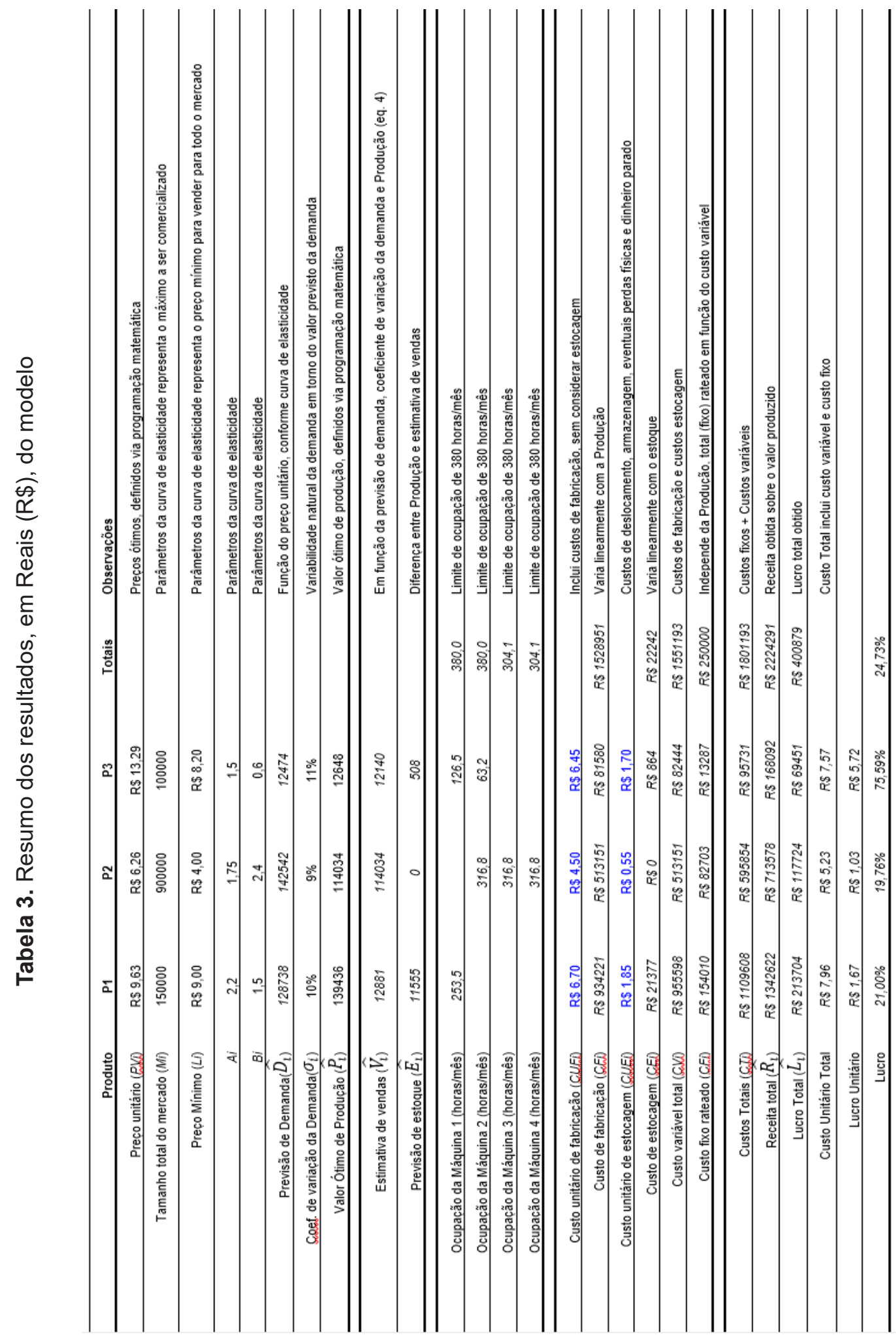


O modelo desenvolvido é aplicado a plantas com múltiplos produtos e múltiplas máquinas, com capacidade finita, características dos ambientes empresariais atuais. Logo, é possível utilizá-lo para analisar a influência de alterações no preço sobre o Lucro global do sistema. Um exemplo desse tipo de análise é apresentado na tabela 4, onde se fixou o preço de venda do produto 1, em um valor diferente do ótimo, deixando que os demais sejam otimizados via programação matemática. É possível perceber que, ao indicar o preço mínimo que absorveria todo o mercado $(\mathrm{L} 1=\mathrm{R} \$ 9,00)$, os valores ótimos de produção e o valor esperado de vendas se aproximam do volume total do mercado $(\mathrm{M} 1=150000 \mathrm{Kg})$.

No entanto, ao comparar os valores obtidos nesta simulação com os resultados obtidos quando todos os preços são otimizados, percebe-se que essa estratégia não é interessante, pois acarretaria em um prejuízo de $22,6 \%$ em relação ao valor máximo do Lucro, obtido quando todos os preços praticados são ótimos.

O mesmo acontece se a empresa decidir realizar um aumento de aproximadamente $10 \%$ no preço do produto 1 , em relação ao preço de venda ótimo. A margem de lucro unitária deste item aumenta em aproximadamente $2 \%$, no entanto o Lucro Total do sistema sofre uma queda de $13,5 \%$.

Logo, é possível perceber a importância do uso de métodos matemáticos de otimização, como o que está proposto neste artigo, para apoiar o tomador de decisão, uma vez que esses métodos consideram as situações reais de mercado, através dos parâmetros inseridos nas curvas de elasticidade preço-demanda, e a capacidade fabril. Outra vantagem do algoritmo proposto é que realizada a modelagem, diversas simulações de interesse podem ser executadas com rapidez, logo, diversos cenários envolvendo, por exemplo, alterações em preço ou nas restrições do sistema produtivo, podem ser analisadas com facilidade.

Tabela 4. Simulação 1 utilizando o modelo proposto

\begin{tabular}{cccccccc}
\hline P1 & $\begin{array}{c}\text { Preço } \\
\text { Unitário }\end{array}$ & $\begin{array}{c}\text { Volume } \\
\text { Produzido }\end{array}$ & $\begin{array}{c}\text { Lucro } \\
\text { Percentual } \\
\text { Unitário }\end{array}$ & $\begin{array}{c}\text { Venda } \\
\text { esperada }\end{array}$ & Lucro Global & $\begin{array}{c}\text { Variação } \\
\text { do Lucro }\end{array}$ \\
\hline $\begin{array}{c}\text { Valor } \\
\text { Otimo }\end{array}$ & R\$ 9,62 & 136628,19 & $21,2 \%$ & 128335,77 & R\$ & 406384,66 & \\
\hline $\begin{array}{c}\text { Valor } \\
\text { Simulado 1 }\end{array}$ & R\$ 9,00 & 153564,83 & $10,8 \%$ & 146579,01 & R\$ & 314502,27 & $-22,6 \%$ \\
\hline $\begin{array}{c}\text { Valor } \\
\text { Simulado 2 }\end{array}$ & R\$ 10,59 & 82370,66 & $23,0 \%$ & 79123,31 & R\$ & 351666,22 & $-13,5 \%$ \\
\hline
\end{tabular}

Outro exemplo que fortalece a importância do uso do modelo pode ser estabelecido alterando o preço do produto 2. Quando é feita uma redução no preço de venda deste produto, em aproximadamente $10 \%$ em relação ao preço ótimo, fixando-se este valor e permitindo que as demais variáveis sejam ajustadas pelo algoritmo, a fim de estabelecer um valor ótimo com esta nova condição, isso ocasiona um aumento de aproximadamente 3,4 vezes no volume demandado. Porém o volume a ser produzido indicado pelo algoritmo permanece próximo dos valores ótimos, o mesmo acontece com a venda esperada. Isso ocorre porque o modelo percebe que, se fosse produzido integralmente o valor demandado, isso estaria associado a margens de lucro muito baixas para este item, mobilizando capacidade produtiva que é melhor utilizada nos demais produtos. Os resultados desse estudo podem ser observados na Tabela 5. 
Tabela 5. Simulação 2 utilizando o modelo proposto

\begin{tabular}{ccccccccc}
\hline P2 & $\begin{array}{c}\text { Preço } \\
\text { Unitário }\end{array}$ & $\begin{array}{c}\text { Volume } \\
\text { Produzido }\end{array}$ & $\begin{array}{c}\text { Previsão } \\
\text { Demanda }\end{array}$ & $\begin{array}{c}\text { Venda } \\
\text { esperada }\end{array}$ & $\begin{array}{c}\text { Lucro } \\
\text { Percentual } \\
\text { Unitário }\end{array}$ & Lucro Global & $\begin{array}{c}\text { Variação } \\
\text { do Lucro }\end{array}$ \\
\hline Valor Ótimo & $\mathrm{R} \$$ & 6,37 & 113114,68 & 114121,27 & 110697,63 & $21,2 \%$ & $\mathrm{R} \$ 406384,66$ & \\
\hline Valor Simulado 1 & $\mathrm{R} \$$ & 5,63 & 113700,39 & 386398,08 & 113700,39 & $7,7 \%$ & $\mathrm{R} \$ 329542,04$ & $-18,9 \%$ \\
\hline
\end{tabular}

Essas análises revelam que mudanças relativamente pequenas nos preços podem ter influência considerável no lucro global do sistema e, portanto, o suporte de modelos matemáticos de otimização deve ser utilizado sempre que possível.

Observa-se que o modelo desenvolvido apresenta diversas vantagens, que serão discutidas a seguir.

Ao estudar o conceito de elasticidade preço-demanda, percebe-se que o mesmo não utiliza variáveis do ambiente externo da organização na sua formulação usual. Mas este modelo, para obter curvas mais próximas da realidade, foram inseridas as variáveis de mercado $M_{i}$, tamanho máximo de mercado para cada produto comercializado, e $L_{i}$, preço de venda mínimo para absorver todo o mercado do produto em análise, as quais são determinadas por especialistas do segmento de mercado em que os produtos são comercializados.

Considera-se como uma vantagem do modelo proposto o tratamento estocástico da demanda, que é modelada em função do preço de venda e sua variabilidade natural, através de uma distribuição de probabilidade construída com base em históricos da quantidade vendida e preços praticados.

Outra vantagem importante deste modelo, pouco explorada na literatura, é a otimização simultânea de preços e volumes de produção associados a cada produto. Além disso, a determinação dos volumes ótimos de produção é feita considerando múltiplos produtos e as capacidades produtivas de múltiplos equipamentos utilizados na fabricação de múltiplos produtos.

A partir das informações de mercado, capacidade fabril e custos, inseridas no algoritmo, como apresentado no método deste artigo, busca-se a otimização do preço de venda e do volume a ser produzido, utilizando o Lucro como função objetivo a ser maximizada. Este objetivo do modelo coincide com o interesse usual das organizações, que visam à maximização do resultado econômico. Logo, o modelo apresenta resultados na linguagem financeira, que oferece maior apoio à gerencia empresarial.

Por fim, uma vez estabelecido, o modelo permite realizar o estudo de diversos cenários rapidamente. Esses cenários podem contemplar alterações nos preços dos produtos (exemplos nas tabelas 1 e 2) ou alterações: na elasticidade preço-demanda dos produtos, na variabilidade da demanda, na capacidade fabril disponível, nos custos de fabricação, nos custos de estocagem e nos custos fixos. 


\section{CONCLUSÃO}

Para que as empresas se mantenham competitivas em seus mercados, as mesmas devem estar constantemente revisando seus planos de produção, apoiadas em estratégias de maximização do resultado econômico. Logo o planejamento de produção deve estar fortemente ligado a área comercial, para realizar o atendimento da demanda considerando as preocupações da gerência empresarial, estabelecendo planos que viabilizem maior lucro para a organização.

Neste estudo, a maximização do resultado foi explorada através de um novo método de planejamento de produção. Esse método envolve a coleta de dados históricos referentes a preços e vendas, a obtenção das curvas de elasticidade preço-demanda, a definição da demanda esperada, da capacidade fabril e dos custos totais de produção. Ele tem como objetivos principais a definição dos preços ótimos de venda e dos volumes ótimos de produção, visando à maximização do Lucro no período. O modelo proposto viabiliza a tomada de decisão baseada em distribuições de probabilidades, aliado a opiniões de especialistas, o que promove maior segurança.

A aplicação do modelo proposto limita-se a indústria de bens manufaturados. Sua principal aplicação está voltada para plantas com múltiplos produtos, múltiplas máquinas e restrições na capacidade fabril, onde a demanda é variável e depende dos preços estabelecidos.

Sugere-se para trabalhos futuros estender o modelo para a cadeia de suprimentos, envolvendo fornecedores de diferentes níveis. O envolvimento de custos e restrições produtivas dos fornecedores propiciará um maior número de variáveis apontadas, maiores possibilidades de ajuste e, consequentemente, maior lucro para o conjunto de empresas envolvidas.

\section{REFERÊNCIAS}

ALMEIDA, A. N.; SILVA, J. C. G. L.; ÂNGELO, H and NUÑEZ, B. E. C. Analysis of factors that influence the log price for mechanical process in the state of Paraná, 2010, Cerne, Lavras, 16 (2), 243-250.

ANTUNES, J.; ALVAREZ, R.; BORTOLOTTO, P.; KLIPPEL, M and PELLEGRIN, I. Sistemas de Produção: Conceitos e Práticas para Projetos para Gestão da Produção Enxuta. Porto Alegre: Bookman, 2008. ISBN 9788577802494.

BILLER, S.; MURIEL, A and ZHANG, Y. Impact of price postponement on capacity and flexibility investment decisions. Production and Operations Management, 2006, 15 (2), 198214.

BOSE, S and PEKNY, J.F.A. Model predictive framework for planning and scheduling problems: a case study of consumer goods supply chain. Computers and Chemical Engineering, 2000, 24, 329-335.

BOTELHO, D. Decomposição da elasticidade-preço no varejo com uso de dados escaneados. Pesquisa Operacional, 2005, 25 (2), 201-221.

CHOD, J and RUDI, N. Strategic investments, trading, and pricing under forecast updating. 
Management Science, 2006, 52 (12), 1913-1929.

DANESE, $P$ and KALCHSCHMIDT, $M$. The role of the forecasting process in improving forecast accuracy and operational performance. International Journal of Production Economics, 2011, 131 (1), 204-214.

ERDIRIK-DOGAN, M and GROSSMANN, I.E. Simultaneous planning and scheduling of single-stage multi-product continuous plants with parallel lines. Computers and Chemical Engineering, 2008, 32, 2664-2683.

FISCHER, M and RAMAN, A. Reducing the cost of demand uncertainty through accurate response to early sales. Operations Research, 1996, 44, 87-99.

HSIEH, C.; LIU, Y and WANG, W. Coordinating ordering and pricing decisions in a two-stage distribution system with price-sensitive demand through short-term discounting. European Journal of Operational Research, 2010, 207, 142-151.

JANS, R.; DEGRAEVE, Z and SCHEPENS, L. Analysis of an industrial component commonality problem. European Journal of Operational Research, 2008, 186, 801-811.

JI, Q.; WANG, Y. and HU, X. Optimal production planning for assembly systems with uncertain capacities and random demand. European Journal of Operational Research, 2016, 253, 383391.

KANNEGIESSER, M.; GÜNTHER, H. O.; BEEK, P. V.; GRUNOW, M. and HABLA, C. Value chain management for commodities : a case study from the chemical industry. Supply Chain Planning, 2009, 1-31.

KAPLAN, U.; TURKAY, M.; KARASOZEN, B and BIEGLER, L. T. Optimization of supply chain systems with price elasticity of demand. INFORMS Journal on Computing, 2010, 23(4),1-12.

KASSEL, S and TITTMANN, C. Implications from customer behavior for manufacturing. Journal of Intelligent Manufacturing, 2007, 18 (4), 475-478.

KIM, D and LEE, W. J. Optimal joint pricing and lot sizing with fixed and variable capacity. European Journal of Operational Research, 1998, 109, 212-227.

LANNING, S.; MITRA, D.; WANG, Q and WRIGHT, M. Optimal planning for optical transport networks. Philosophical Transactions of the Royal Society of London A: Mathematical, Physical and Engineering Sciences, 2000, 358 (1773), 2183-2196.

LEUNG, K. F. A generalized geometric-programming solution to "An economic production quantity model with flexibility and reliability considerations". European Journal of Operational Research, 2007, 176, 240-251.

LEVIS, A. A and PAPAGEORGIOU, L. G. Active demand management for substitute products through price optimization. OR Spectrum, 2007, 29, 551-577.

LINGITZ, L.; MORATEWETZ, C.; GIGLOO, D. T.; MINNER, S and SIHN, W. Modelling of flexibility costs in a decision support system for midterm capacity planning. Procedia CIRP 7 , 46th CIRP Conference on Manufacturing Systems, 2013, 539-544. 
LIU, S.; SHAH, N and PAPAGEORGIOU, L. G. Multiechelon Supply Chain Planning with Sequence-Dependent Changeovers and Price Elasticity of Demand under Uncertainty. Wiley Online Library, 2012, 58 (11), 3390-3403.

MARTÍNEZ-COSTA, C.; MAS-MACHUCA, M.; BENEDITO, E and COROMINAS, A. A review of mathematical programming models for strategic capacity planning in manufacturing. Int. J. Production Economics, 2014, 153, 66-85.

MORATEWETZ, C and SIHN, W. Model of a Decision Support System for a Least-Cost and Harmonized Capacity Adjustment in the Short- and Medium-Term Planning Horizon. Procedia CIRP 3, 45th CIRP Conference on Manufacturing Systems, 2012, 20-25.

PAPAGEORGIOU, L.G. Supply chain optimization for the process industries: Advances and opportunities. Computers and Chemical Engineering, 2009, 33, 1931-1938.

PEREA-LO, E.; YDSTIE, B. E and GROSSMANN, I. E. A model predictive control strategy for supply chain optimization. Computers and Chemical Engineering, 2003, 27, 1201-1218.

SADJADI, S. J.; YAZDIAN, S. A and SHAHANAGHI, K. Optimal pricing, lot-sizing and marketing planning in a capacitated and imperfect production system. Computers and Industrial Engineering, 2012, 62, 349-358.

SEN, W.; POKHAREL, S and YULEI, W. Supply chain positioning strategy integration, evaluation, simulation, and optimization. Computers and Industrial Engineering, 2004, 46, 781-792.

SHAPIRO, J. F.; Challenges of strategic supply chain planning and modeling. Computers and Chemical Engineering, 2004, 28, 855-861.

SILVA, E. L and MENEZES, E. M. Metodologia da pesquisa e elaboração de dissertação. Florianópolis: Laboratório de Ensino à Distância da UFSC, 3 ed. 2001.

SUBRAMANIAN, K.; RAWLINGS, J. B.; MARAVELIAS, C. T and FLORES-CERRILLO, J.; MEGAN, L. Integration of control theory and scheduling methods for supply chain Management. Computers and Chemical Engineering, 2013, 51, 4-20.

TAN, T and ALP, O. An integrated approach to inventory and flexible capacity management subject to fixed costs and non-stationary stochastic demand. OR Spectrum, 2009, 31, 337360.

TELLIS, G. J. The price elasticity of selective demand: A meta-analysis of econometric models of sales. Journal of Marketing Research, 1988, 25, 331-341.

VISWANATHAN, S and WANG Q. Discount pricing decisions in distribution channels with price-sensitive demand. European Journal of Operational Research, 2003, 149, 571-587.

ZHANG, B.; HU, S.; SONG, J and CHENG, S. Analysis about Medium-long-term Demands Forecasting and Capacity Decision Based on the Grey $\mathrm{GM}(1,1)$ Improved Models. International Conference on Management Science \& Engineering, 2012, 19, 20-22. 